\title{
Net emission coefficients of argon iron plasmas with electron Stark widths scaled to experiments
}

\author{
M. Wendt \\ INP Greifswald, Leibniz Institute for Plasma Science and Technology, \\ Felix-Hausdorff-Str. 2, 17489 Greifswald, Germany \\ E-mail: wendt@inp-greifswald.de
}

\begin{abstract}
.
The net emission coefficient of plasmas containing argon and iron at atmospheric pressure is calculated and analysed for the case of cylindrical geometry. Its values are obtained by integrating the monochromatic net emission coefficient taking into account continuous and line radiation. The width of the spectral lines is determined by Doppler broadening, natural, resonance, van der Waals, electron and ion Stark broadening. As Stark broadening is the most important broadening mechanism in the considered pressure and temperature range, the electron Stark widths are calculated following the semi-empirical Stark broadening theory. Additionally, the electron Stark widths of $\mathrm{Ar}, \mathrm{Ar}^{+}, \mathrm{Fe}$ and $\mathrm{Fe}^{+}$are multiplied by scaling factors in order to reproduce experimental electron Stark widths. The scaling factor is determined for each species separately. For small plasma radii the net emission coefficient determined here shows good agreement with literature values where spherical geometry is considered while they decrease faster with increasing plasma radius. This behaviour is caused by the increase of the irradiation of the symmetry axis when cylindrical instead of spherical geometry is considered. For radii and temperatures typical of the metal filled core of arcs occurring in gas metal arc welding processes, i.e. radii between 1 and $2 \times 10^{-3} \mathrm{~m}$ and temperatures between 5000 and $10000 \mathrm{~K}$, the scaling of the Stark widths increases the net emission coefficient of iron plasmas by between 2 and $23 \%$. In this parameter range the net emission coefficient of iron plasmas for cylindrical geometry is between 30 and $37 \%$ smaller than values calculated for spherical geometry.
\end{abstract}

PACS numbers: 52.25.Os, 52.77.Fv, 81.20.Vj

Submitted to: J. Phys. D: Appl. Phys. 


\section{Introduction}

In atmospheric pressure arcs such as welding arcs $[1,2]$ the energy transport by radiation has a significant influence on the energy balance and needs to be included in corresponding numerical models. The conditions in these arcs are such that the plasma is optically thick in a significant range of wavelengths so that a treatment of the radiation transport has to take into account reabsorption. An exact treatment of the radiation transport based on the radiation transport equation leads to high numerical costs, because application relevant species like iron show a multitude of spectral lines. In numerical models of atmospheric pressure arcs the exact radiation transport term is consequently often replaced by the net emission coefficient as introduced by Lowke [3]. Thus net emission coefficients remain important input data for numerical modelling of welding arcs as done by e.g. Lowke et al [4] and Schnick et al [5]. The use of net emission coefficients in such models has the drawback that it does not take into account the inhomogeneity of the temperature and the spatial distribution of the elements in experimental arcs. More advanced approximations of the radiation transport equation are discussed by e.g. Modest [6].

Net emission coefficients of argon iron plasmas have been calculated by Cram [7], Essoltani et al [8], Gleizes et al [9], and Menart et al [10]. Net emission coefficients for iron-helium plasmas have been calculated by Moscicki et al [11].

Cram [7] considers line radiation only. In this investigation the total number of lines is estimated from a subset of spectral lines by means of a statistical method [12], so that the need for extensive line data tabulations is eliminated. The only considered broadening mechanism is Stark broadening, and absorption is accounted for by means of line escape factors $[13,14]$. Essoltani et al [8] use line data tables from the National Institute of Standards and Technology (NIST), and include bound-free and free-free radiation. The line broadening takes account for natural, resonance, van der Waals, Stark and Doppler broadening while absorption is treated by line escape factors. Gleizes et al [9] also consider line and continuous radiation processes using improved line data tables from NIST and additional data from Kurucz and Peytremann [15]. Doppler broadening and pressure broadening are considered. Absorption of lines with high emissivity is treated using escape factors while lines with low emissivity and the continuum are considered as optically thin. Menart et al [10] take account for both line and continuum radiation. Their results are based on line data tables from NIST and the Kurucz database. Extrapolation procedures are employed to add missing atomic levels and lines so that these authors include still more spectral lines than Gleizes et al [9]. The net emission coefficients are calculated by integrating the monochromatic net emission coefficient. Moscicki et al [11] also include line and continuum radiation. Their results are based on line data tables given by Bautista [16] and Nahar [17]. These tables of theoretical transition probabilities provide approximately the same number of lines for neutral iron as used by Menart et al [10] while the number of lines given for singly and doubly ionized iron ions is approximately a factor of two smaller. 
Essoltani et al and Gleizes et al estimate the electron Stark widths via classical formulae [18]. The present work follows the more accurate method used by Menart et al $[10,19]$. Menart et al calculate electron Stark widths using the semi-empirical theories of Griem [20], and Dimitrijević and Konjević [21,22]. These theories take into account quantum mechanical effects and inelastic collisions between the radiator and electrons. In order to use electron Stark widths that closely reproduce experimental electron Stark widths, a scaling factor is introduced for each species where experimental electron Stark widths are available. While this procedure is applied to argon and copper in [19] it is not mentioned in [10] so that to our knowledge it is first applied to iron here.

The earlier investigations on argon iron plasmas [8-11] consider an isothermal, homogenous plasma of spherical shape while this work assumes cylindrical geometry. This change of geometry leads to an increased irradiation at the symmetry axis so that the net emission coefficient is reduced. The net emission coefficients presented below are based on the monochromatic emission coefficient for argon iron plasmas assuming local thermodynamic equilibrium (LTE). The monochromatic emission coefficient includes line radiation, free-bound and free-free transitions. The spectral lines are broadened by natural, resonance, van der Waals, electron and ion Stark broadening and by Doppler broadening.

\section{Theory}

The net emission coefficient $\epsilon_{\text {net }}$ is defined as the power lost by radiation per volume and solid angle, that is

$$
\epsilon_{\mathrm{net}}=\int_{\lambda}\left(\epsilon_{\lambda}-\kappa^{\prime} J_{\lambda}\right) \mathrm{d} \lambda
$$

where $\epsilon_{\lambda}$ is the monochromatic emission coefficient per wavelength $\lambda, \kappa^{\prime}$ the monochromatic absorption coefficient including induced emission and

$$
J_{\lambda}=\frac{1}{4 \pi} \int_{\Omega} L_{\lambda} \mathrm{d} \Omega
$$

the solid angle averaged spectral radiance impinging onto the considered point in the plasma. On the axis of a homogeneous, cylindrical plasma column of radius $R$ it holds

$$
\epsilon_{\mathrm{net}}(R, T)=\int_{\lambda} \epsilon_{\lambda}(T) \mathrm{G}_{1}\left(\kappa^{\prime} R\right) \mathrm{d} \lambda
$$

where $G_{1}$ is a function taking account for the considered geometry. For cylindrical geometry it holds [23]

$$
\mathrm{G}_{1}(x)=\int_{0}^{\pi / 2} \exp \left(-\frac{x}{\sin \phi}\right) \sin \phi \mathrm{d} \phi .
$$

It can be shown that $\mathrm{G}_{1}$ is identical to $\mathrm{Ki}_{2}$, the second integral of the modified Bessel function $\mathrm{K}_{0}$ [24]. It has the limits $1-\pi x / 2$ and $\sqrt{\pi /(2 x)} \exp (-x)$ for small and for large $x$, respectively. While Lowke [3] evaluated $\mathrm{G}_{1}(x)$ by a numerical treatment of 
(4), Lee [25] used series expansions based on modified Bessel functions. For spherical geometry it holds $\mathrm{G}_{1}(x)=\exp (-x)$.

In local thermodynamic equilibrium the monochromatic emission and absorption coefficients obey Kirchhoff's law

$$
\epsilon_{\lambda}=\kappa^{\prime} L_{\lambda}^{\mathrm{Pl}}
$$

where

$$
L_{\lambda}^{\mathrm{Pl}}=\frac{2 h c^{2}}{\lambda^{5}} /\left[\exp \left(\frac{h c}{\lambda k T}\right)-1\right]
$$

is the black body spectral radiance with $h, c, k$, and $T$ being the Planck constant, the velocity of light, the Boltzmann constant and the temperature, respectively. In (3) Kirchhoff's law is used to express the absorption coefficient by the emissions coefficient.

The total monochromatic emission coefficient $\epsilon_{\lambda}$ is the sum of the species emission coefficients $\epsilon_{\lambda, r}$ where $r$ denotes the atomic and ionic species of the plasma. As the emission coefficients $\epsilon_{\lambda, r}$ are proportional to the species densities $N_{r}$, the plasma composition needs to be known. The plasma composition is calculated assuming LTE taking weakly non-ideal corrections due to the Coulomb interaction into account [26]. The composition includes the species $\mathrm{Ar}, \mathrm{Ar}^{+}, \mathrm{Ar}^{2+}, \mathrm{Fe}, \mathrm{Fe}^{+}, \mathrm{Fe}^{2+}$, and electrons. The definition of the iron mole fraction $x_{\mathrm{Fe}}$, the equation of state and the Saha equation for stepwise ionization thus read

$$
\begin{aligned}
& x_{\mathrm{Fe}}=\frac{N_{\mathrm{Fe}}+N_{\mathrm{Fe}^{+}}+N_{\mathrm{Fe}^{2+}}}{N_{\mathrm{Fe}}+N_{\mathrm{Fe}^{+}}+N_{\mathrm{Fe}^{2+}}+N_{\mathrm{Ar}}+N_{\mathrm{Ar}^{+}}+N_{\mathrm{Ar}^{2+}}} \\
& P=k T \sum_{r} N_{r}+k T N_{\mathrm{e}}+P^{\mathrm{Co}} \\
& \frac{N_{\mathrm{e}} N_{z+1}}{N_{z}}=2 \frac{Z_{z+1}}{Z_{z}} \frac{\Lambda_{z}^{3}}{\Lambda_{\mathrm{e}}^{3} \Lambda_{z+1}^{3}} \mathrm{e}^{-\left(I_{z}+\mu_{\mathrm{e}}^{\mathrm{Co}}+\mu_{z+1}^{\mathrm{Co}}-\mu_{z}^{\mathrm{Co}}\right) /(k T)}
\end{aligned}
$$

where $P, N_{\mathrm{e}}, P^{\mathrm{Co}}, \mu_{\mathrm{e}}^{\mathrm{Co}}$ and $\mu_{\mathrm{r}}^{\mathrm{Co}}$ are the total pressure, the electron density, the Coulombpressure, the non-ideal chemical potentials of the electrons, and the non-ideal chemical potentials of atoms or ions, respectively. Indices $z$ and $z+1$ denote an atomic or ionic species with charge number $z$ and its ion of the next ionization step, respectively. In (9)

$$
Z_{r}(T)=\sum_{r} g_{i, r}\left[\mathrm{e}^{-E_{i, r} / k T}+\mathrm{e}^{-I_{r} / k T}\left(\frac{E_{i, r}-I_{r}}{k T}-1\right)\right]
$$

is the Planck-Larkin partition function of species $r$ with ionization energy $I_{r}$, internal state energies $0 \leq E_{i, r}<I_{r}$, and statistical weights $g_{i, r}$, and $\Lambda_{k}=h\left(2 \pi m_{k} k T\right)^{-1 / 2}$ with $k=r$ or e is the thermal DeBroglie wavelength of species $k$ with mass $m_{k}$. The Planck-Larkin partition function arises from the quantum statistical theory presented in [26]. Using the Debye-Hückel screened potential with minimum contact distance $\Lambda_{\mathrm{e}} / 8$ leads to the non-ideal terms

$$
\begin{aligned}
& P^{\mathrm{Co}}=-\frac{128 k T}{\pi \Lambda_{\mathrm{e}}^{3}}\left[\frac{q(q+2)}{2(q+1)}-\log (1+q)\right] \\
& \mu_{k}^{\mathrm{Co}}=-\frac{\left(e z_{k}\right)^{2}}{8 \pi \epsilon_{0} r_{\mathrm{D}}} \frac{1}{1+q}
\end{aligned}
$$


where $e$ is the elementary charge, $\epsilon_{0}$ is the permittivity of free space, $k=r$ or e,

$$
r_{\mathrm{D}}=\left(\frac{\epsilon_{0} k T}{e^{2}\left(N_{\mathrm{e}}+\sum_{r} z_{r}^{2} N_{r}\right)}\right)^{1 / 2}
$$

is the Debye length and $q=\Lambda_{\mathrm{e}} /\left(8 r_{\mathrm{D}}\right)$. The non-ideal chemical potentials $\mu_{k}^{\text {Co }}$ lead to an apparent reduction of the ionization energies. For the plasmas considered here the above non-ideal corrections remain small. For pure argon and pure iron plasmas they lead to a maximum increase of the electron density by 8 and $13 \%$, respectively.

\subsection{Emission coefficients}

For each atom or ion $r$ the monochromatic species emission coefficient is given by

$$
\epsilon_{\lambda, r}=\epsilon_{\lambda, r}^{\mathrm{bb}}+\epsilon_{\lambda, r}^{\mathrm{fb}}+\epsilon_{\lambda, r}^{\mathrm{ff}}
$$

where the right hand side terms represent bound-bound, free-bound and free-free transitions corresponding to line radiation, radiation from the recombination continuum and bremsstrahlung, respectively. For each of these processes a corresponding induced emission process and an absorption process exists. The treatment of each emission process is described below. The subscript $\lambda$ denotes that a spectral quantity is given per wavelength unit.

The emission coefficient for line radiation of atomic or ionic species $r$ is given by

$$
\epsilon_{\lambda, r}^{\mathrm{bb}}=\sum_{u l} \frac{h c}{4 \pi \lambda_{u l}} A_{u l} N_{u} P_{\lambda}(\lambda)
$$

where $\lambda_{u l}, A_{u l}, N_{u}$, and $P_{\lambda}(\lambda)$ are the wavelength of the transition from upper state $u$ to lower state $l$, the transition probability, the upper state density and the line profile function, respectively. The index $u l$ on $P_{\lambda}(\lambda)$ has been dropped. The width and shape of the profile function depends on temperature and the plasma composition and has to be calculated for each line separately. The line profile function used here is described in section 2.2 .

The emission due to the recombination of ions and electrons forming species $r$ is given by the emission coefficient [27]

$$
\epsilon_{\lambda, r}^{\mathrm{fb}}=\frac{N_{r}}{Z_{r}} \frac{2 h c^{2}}{\lambda^{5}} \mathrm{e}^{-h c /(\lambda k T)} \sum_{i} \sigma_{i, r}(\lambda) g_{i, r} \mathrm{e}^{-E_{i, r} /(k T)} .
$$

Here, $\sigma_{i, r}$ is the photoionization cross section of internal state $i$. For excited states the semiclassical photoionization cross section [27]

$$
\sigma_{i, r}(\lambda)=\frac{64 \pi}{3 \sqrt{3}} a_{0}^{2} \alpha_{\mathrm{f}}\left(z_{r}+1\right)^{4}\left(\frac{E_{\mathrm{H}}}{h c}\right)^{3} \frac{\lambda^{3}}{n_{i, r}^{* 5}}
$$

is used. Here, $a_{0}, \alpha_{\mathrm{f}}, E_{\mathrm{H}}$ and $n_{i, r}^{*}=\left[E_{\mathrm{H}} /\left(I_{r}-E_{i, r}\right)\right]^{1 / 2}$ are the Bohr radius, the fine structure constant, the Rydberg energy and the effective main quantum number of state $i$. For wavelengths above $h c /\left(I_{r}-E_{i, r}\right)$ the photoionization cross section is zero.

For the ground states of argon, iron and their ions photoionization cross sections are used which are more accurate than those given by (17). Following [28] theoretical 
Table 1. Parameters of the photoionization cross sections for atomic and ionic ground states as taken from [30] for argon and from [35] for the energy region for iron species, a) $1.709+1$ denotes $1.709 \times 10^{1}$.

\begin{tabular}{lccccccc}
\hline & $\begin{array}{c}E_{0} \\
\mathrm{eV}\end{array}$ & $\begin{array}{c}\sigma_{0} \\
10^{-22} \mathrm{~m}^{2}\end{array}$ & $y_{\mathrm{a}}$ & $P$ & $y_{\mathrm{w}}$ & $y_{0}$ & $y_{1}$ \\
\hline $\mathrm{Ar}$ & $1.709+1^{\mathrm{a})}$ & $2.106+1$ & $2.645+2$ & $4.706+0$ & $4.185-1$ & $1.688+0$ & $8.943-1$ \\
$\mathrm{Ar}^{+}$ & $2.494+1$ & $2.503+1$ & $1.272+2$ & $4.288+0$ & $5.108-1$ & $9.299-1$ & $7.195-1$ \\
$\mathrm{Ar}^{2+}$ & $1.417+1$ & $3.580+1$ & $3.776+1$ & $5.742+0$ & $6.316-1$ & $2.384+0$ & $1.794+0$ \\
$\mathrm{Fe}$ & $1.407+2$ & $1.083+4$ & $4.458+0$ & $1.691+1$ & $4.039-1$ & $0.000+0$ & $0.000-0$ \\
$\mathrm{Fe}^{+}$ & $1.933+1$ & $3.679+4$ & $3.564+0$ & $1.566+1$ & $4.144-1$ & $0.000+0$ & $0.000+0$ \\
$\mathrm{Fe}^{2+}$ & $2.670+1$ & $6.301+3$ & $5.385+0$ & $1.232+1$ & $4.407-1$ & $0.000+0$ & $0.000+0$ \\
\hline
\end{tabular}

photoionization cross sections from the Opacity Project (OP) [29] are employed for the ground states of argon and its ions. Verner et al [30] smoothed these cross sections and presented them in the form

$$
\sigma(E)=\sigma_{0} y^{(P-11-2 \ell) / 2}\left[(x-1)^{2}+y_{\mathrm{w}}^{2}\right]\left(1+\sqrt{\frac{y}{y_{\mathrm{a}}}}\right)^{-P}
$$

using the abbreviations $x=E / E_{0}-y_{0}$ and $y=\left(x^{2}+y_{1}^{2}\right)^{1 / 2}$. Here, $\sigma_{0}, E_{0}, y_{0}, y_{1}, y_{\mathrm{a}}$, $y_{\mathrm{w}}$ and $P$ are fit parameters (Table 1$), \ell$ is the orbital quantum number of the photo electron and $E=h c / \lambda$ is the photon energy. For neutral argon the photoionization cross section given in [30] is in good agreement with the recommended experimental values given by Marr and West [31].

For iron and its ions the photoionization cross sections given in $[32,33]$ and $[34]$ are used. Following the approach of Verner et al [30] the autoionization resonances contained in these cross sections are smoothed using a Gauss profile with a half width at half maximum of $5 \mathrm{~nm}$. For high photon energies where cross sections are not given in the OP data, cross sections from Verner et al [35] are employed. These cross sections are best fits to theoretical cross sections calculated using the Hartree-Dirac-Slater method which provides photoionization cross sections with correct non-relativistic high energy asymptotes. They are also given in the form (18). While the OP photoionization cross sections for iron take account for ionization from both the $4 \mathrm{~s}$ and the $3 \mathrm{~d}$ subshell, the high energy photoionization cross sections of Verner et al [35] treat each subshell separately. The cross sections of the $3 \mathrm{~d}$ subshell $(\ell=2)$ are chosen here because they are approximately one order of magnitude higher than those of the 4 s subshell.

The emission due to free-free transitions of electrons in the electric field of ions of species $r$ is treated by the emission coefficient [27]

$$
\epsilon_{\lambda, r}^{\mathrm{ff}}=\frac{16 \pi}{3 \sqrt{3}}\left(\frac{e^{2}}{4 \pi \epsilon_{0}}\right)^{3} \frac{z_{r}^{2}}{\left(\lambda m_{\mathrm{e}} c\right)^{2}} \sqrt{\frac{m_{\mathrm{e}}}{2 \pi k T}} \mathrm{e}^{-h c /(\lambda k T)} N_{\mathrm{e}} N_{r} .
$$


The free-free radiation emitted in collisions of electrons with neutral species $r$ is described by $[36,37]$

$$
\epsilon_{\lambda, r}^{\mathrm{ff}}=\frac{h c}{4 \pi \lambda^{2}} N_{\mathrm{e}} N_{r} \int_{E=h c / \lambda}^{\infty} Q(\lambda, E) \sqrt{\frac{4}{\pi m_{\mathrm{e}} k T}} \frac{E}{k T} \mathrm{e}^{-E /(k T)} \mathrm{d} E
$$

with

$$
Q(\lambda, E)=\left(1-\frac{h c}{2 \lambda E}\right)\left(1-\frac{h c}{\lambda E}\right)^{1 / 2} \frac{8 \alpha_{\mathrm{f}}}{3 \pi} \frac{E}{m_{\mathrm{e}} c^{2}} Q^{\mathrm{mom}}(E) .
$$

In these expressions $E$ is the kinetic energy of the electron, and $Q^{\text {mom }}$ the momentum transfer cross section of the considered atom. In this work the momentum transfer cross sections given in [38] is used for Ar. For Fe the polarization cross section $Q(E)=4 \pi A\left[\alpha e^{2} /\left(4 \pi \epsilon_{0} E\right)\right]^{1 / 2}$ with $A=0.6037[39]$ and the polarization volume $\alpha=8.4 \times 10^{-30} \mathrm{~m}^{3}[40]$ is employed to approximate its momentum transfer cross section for elastic collisions with electrons.

\subsection{Line profile function}

In typical welding plasmas spectral lines are subject to natural, resonance, van der Waals, and Stark broadening. For typical temperatures van der Waals and Stark broadening can be treated in the impact approximation so that these broadening mechanisms lead to a Lorentz line profile with a half widths at half maximum (HWHM)

$$
\Delta \lambda=\Delta \lambda_{\text {nat }}+\Delta \lambda_{\text {res }}+\Delta \lambda_{\mathrm{vdW}}+\Delta \lambda_{\mathrm{S}}
$$

where $\Delta \lambda_{\text {nat }}, \Delta \lambda_{\text {res }}, \Delta \lambda_{\mathrm{vdW}}$, and $\Delta \lambda_{\mathrm{S}}$ are the half widths of natural, resonance, van der Waals and Stark broadening, respectively. Additionally, the spectral lines are broadened by the Doppler effect showing a Gauss line profile with width

$$
\Delta \lambda_{\mathrm{Dpl}}=\lambda_{u l}\left(2 \ln 2 \frac{k T}{m_{r} c^{2}}\right)^{1 / 2} .
$$

The total line profile is thus a Voigt profile. In this work it is treated by means of the numerical approximation given by Whiting [41].

The natural width $\Delta \lambda_{\text {nat }}$ of a line is given by the life times of its upper and lower states. Considering only spontaneous emission the natural line width is given by [18]

$$
\Delta \lambda_{\text {nat }}=\frac{\lambda_{u l}^{2}}{4 \pi c}\left(\sum_{u^{\prime}<u} A_{u u^{\prime}}+\sum_{l^{\prime}<l} A_{l l^{\prime}}\right) .
$$

Here, $u^{\prime}<u$ designates states $u^{\prime}$ that are connected to the upper state $u$ by a dipole transition and have an energy $E_{u^{\prime}}$ smaller than $E_{u}$. Analogously, $l^{\prime}<l$ designates states $l^{\prime}$ with $E_{l^{\prime}}<E_{l}$.

Resonance broadening occurs in collisions where radiator and perturber species are identical and if a dipole transition exists between upper or lower level of the considered line and the state of the perturber. The line width due to one such so called resonant transition is [18]

$$
\Delta \lambda_{\text {res }}=\frac{\lambda_{u l}^{2}}{2 \pi c} \frac{5.48}{64 \pi^{2}} \sqrt{\frac{g_{u \mathrm{R}}}{g_{l \mathrm{R}}}} A_{u l \mathrm{R}} \lambda_{\mathrm{R}}^{3} N_{p}
$$


where $g_{u \mathrm{R}}, g_{l \mathrm{R}}, A_{u l \mathrm{R}}, \lambda_{\mathrm{R}}$, and $N_{p}$ are the statistical weights, the transition probability and the wavelength of the resonant transition and the perturber state density, respectively. Considering the collisions of the radiator with different perturber states as statistically independent, an extension of (25) to all possible perturber states reads

$$
\begin{aligned}
\Delta \lambda_{\text {res }}= & \frac{\lambda_{u l}^{2}}{2 \pi c} \frac{5.48}{64 \pi^{2}} \frac{N_{r}}{Z_{r}} \times \\
& \left(\sum_{u^{\prime}>u} \sqrt{\frac{g_{u^{\prime}}}{g_{u}}} g_{u^{\prime}} A_{u^{\prime} u} \lambda_{u^{\prime} u}^{3} \mathrm{e}^{-E_{u^{\prime}} / k T}+\sum_{u^{\prime}<u} \sqrt{\frac{g_{u}}{g_{u^{\prime}}}} g_{u^{\prime}} A_{u u^{\prime}} \lambda_{u u^{\prime}}^{3} \mathrm{e}^{-E_{u^{\prime}} / k T}\right. \\
& \left.+\sum_{l^{\prime}>l} \sqrt{\frac{g_{l^{\prime}}}{g_{l}}} g_{l^{\prime}} A_{l^{\prime} l} \lambda_{l^{\prime} l}^{3} \mathrm{e}^{-E_{l^{\prime}} / k T}+\sum_{l^{\prime}<l} \sqrt{\frac{g_{l}}{g_{l^{\prime}}}} g_{l^{\prime}} A_{l l^{\prime}} \lambda_{l l^{\prime}}^{3} \mathrm{e}^{-E_{l^{\prime}} / k T}\right)
\end{aligned}
$$

While this sum is often dominated by the resonant state with the lowest energy, this state does not have to be the ground state of the radiator, e.g. for many lines of neutral iron the lowest resonant state is an excited state within the ground term $a^{5} D$.

The lines of neutral radiators are subject to van der Waals broadening due to collisions with neutrals. The corresponding line width is [42]

$$
\Delta \lambda_{\mathrm{vdW}}=\frac{\lambda_{u l}^{2}}{2 \pi c} 4.24\left|C_{6, u}-C_{6, l}\right|^{2 / 5} \sum_{z_{p}=0} \alpha_{p}^{2 / 5}\left(\frac{8 k T}{\pi \mu_{p}}\right)^{3 / 10} N_{p}
$$

where $\alpha_{p}$ is the polarization volume of perturber $p, \mu_{p}=m_{p} m_{r} /\left(m_{p}+m_{r}\right)$ is the reduced mass of the radiator perturber system and the interaction constant $C_{6, i}$ is given by [42]

$$
C_{6, i}=\frac{h a_{0}}{2 \pi m_{\mathrm{e}}} \frac{\left(n_{i, r}^{*}\right)^{2}}{2}\left[5\left(n_{i, r}^{*}\right)^{2}+1-3 \ell_{i, r}\left(\ell_{i, r}+1\right)\right]
$$

where $\ell_{i, r}$ is the orbital quantum number of the radiating electron in state $i$.

Lines of neutral and charged species are subject to Stark broadening by electrons and by ions leading to line widths $\Delta \lambda_{\mathrm{Se}}$ and $\Delta \lambda_{\mathrm{Si}}$, respectively. For typical parameters of atmospheric plasmas the impact approximation can be used for electron Stark broadening while the quasi-static approximation has to be applied to the Stark broadening by ions. Griem showed that in this case the total line profile for Stark broadening can in good approximation be described by a Lorentz profile with the effective width

$$
\Delta \lambda_{\mathrm{S}}=\Delta \lambda_{\mathrm{Se}}[1+1.75 A(1-\xi R)]
$$

where $\xi=0.75$ for neutral radiators and $\xi=1.25$ for charged radiators [43]. Here [44],

$$
A=\left(\frac{F_{0}^{2}}{2 h c} \frac{\lambda_{u l}^{2}}{\Delta \lambda_{\mathrm{Se}}}\left|\alpha_{u}-\alpha_{l}\right|\right)^{3 / 4}
$$

is the ion broadening parameter of the line,

$$
F_{0}=\frac{1}{2}\left(\frac{4}{15}\right)^{2 / 3} \frac{e}{\epsilon_{0}} \sum_{j \neq \mathrm{e}} z_{j} N_{j}^{2 / 3}
$$


is the normal field strength,

$$
\alpha_{i}=\frac{2}{3 \pi}\left(\frac{h^{2} \epsilon_{0}}{m_{\mathrm{e}} e}\right)^{2} \sum_{i^{\prime}} \frac{R_{i i^{\prime}}^{2}}{\Delta E_{i i^{\prime}}}
$$

is the polarizability of state $i, \Delta E_{i i^{\prime}}=\left|E_{i}-E_{i^{\prime}}\right|$ is the energy separation of states $i$ and $i^{\prime}$ and $R_{i i^{\prime}}$ is the coordinate operator matrix element between these states, as described further below. The parameter

$$
R=\frac{r_{p}}{r_{\mathrm{D}}}=\left(\frac{4 \pi}{3} \sum_{z_{j}>0} N_{j}\right)^{-1 / 3} \frac{1}{r_{\mathrm{D}}}
$$

is the ratio of the mean ion-ion distance $r_{p}$ and the Debye length $r_{\mathrm{D}}$. The approximation (29) can be applied for $0.05 \leq A \leq 0.5$ and $R \leq 0.8$. For $A<0.05$ the total Stark broadening width has to be calculated by

$$
\Delta \lambda_{\mathrm{S}}=\Delta \lambda_{\mathrm{Se}}+\Delta \lambda_{\mathrm{Si}}
$$

where

$$
\Delta \lambda_{\mathrm{Si}}=\frac{\lambda_{u l}^{2}}{2 \pi c} \frac{h a_{0}}{2 \pi m_{\mathrm{e}}}\left(\frac{n_{u, r}^{* 2}-n_{l, r}^{* 2}}{z_{r}+1}\right)^{2} \sum_{z_{p}>0} z_{p} N_{p}
$$

is the width of quadrupole ion broadening. For $A>0.5$ a transition to linear Stark broadening occurs [43]. In the approach used here the ion Stark broadening widths are set to the maximum of $\Delta \lambda_{\mathrm{Si}}$ and $1.75 A(1-\xi R) \Delta \lambda_{\mathrm{Se}}$. For $R>0.8$ or $A>0.5$ the total Stark width $(29)$ is evaluated at the corresponding boundary value.

The electron Stark broadening has to be treated differently for neutral atoms, singly and multiply charged ions. Dimitrijević and Konjević [21] give the following formulae for the electron Stark broadening of lines of neutral atoms

$$
\begin{aligned}
\Delta \lambda_{\mathrm{Se}}= & s_{r} N_{\mathrm{e}} \frac{\lambda_{u l}^{2}}{2 \pi c} \frac{h a_{0}}{m_{\mathrm{e}}} \sqrt{\frac{8}{27} \frac{E_{\mathrm{H}}}{k T}} \\
& \times\left[\sum_{u^{\prime}} R_{u u^{\prime}}^{2} f_{\mathrm{w}}\left(\eta_{u u^{\prime}} R_{u u^{\prime}}\right)+\sum_{l^{\prime}} R_{l l^{\prime}}^{2} f_{\mathrm{w}}\left(\eta_{l l^{\prime}} R_{l l^{\prime}}\right)\right]
\end{aligned}
$$

with the definition $\eta_{a b}=\Delta E_{a b} /(3 k T)$ and the effective Gaunt factor

$$
f_{\mathrm{w}}(x)=\mathrm{e}^{-1.33 x} \ln \left(1+\frac{2.27}{x}\right)+\frac{0.487 x}{0.153+x^{5 / 3}}+\frac{x}{7.93+x^{3}} .
$$

In (36) the scaling factor $s_{r}$ for species $r$ is introduced. This factor is used to scale the theoretical Stark widths of each neutral and ion species to measured Stark widths found in the literature.

For singly charged positive ions Griem [20] gives the formula

$$
\begin{aligned}
\Delta \lambda_{\mathrm{Se}}= & s_{r} N_{\mathrm{e}} \frac{\lambda_{u l}^{2}}{2 \pi c} \frac{h a_{0}}{m_{\mathrm{e}}} \sqrt{\frac{16 \pi}{27} \frac{E_{\mathrm{H}}}{k T}} \\
& \times\left[\sum_{u^{\prime}} R_{u u^{\prime}}^{2} g_{\mathrm{se}}\left(\frac{3 k T}{2 \Delta E_{u u^{\prime}}}\right)+\sum_{l^{\prime}} R_{l l^{\prime}}^{2} g_{\mathrm{se}}\left(\frac{3 k T}{2 \Delta E_{l l^{\prime}}}\right)\right]
\end{aligned}
$$


where $g_{\text {se }}$ is an effective Gaunt factor plotted in [20].

Doubly and higher charged ions are treated according to Dimitrijević and Konjević [22] using

$$
\begin{aligned}
& \Delta \lambda_{\mathrm{Se}}=s_{r} N_{\mathrm{e}} \frac{\lambda_{u l}^{2}}{2 \pi c} \frac{h a_{0}}{m_{\mathrm{e}}} \sqrt{\frac{16 \pi}{27} \frac{E_{\mathrm{H}}}{k T}} \times[ \\
& \sum_{u^{\prime}} R_{u u^{\prime} \mid \Delta n \neq 0}^{2} g_{\text {se }}\left(\frac{3 k T n_{u, r}^{*}{ }^{3}}{4 E_{\mathrm{H}} z_{r}^{2}}\right)+\sum_{l^{\prime}} R_{l l^{\prime} \mid \Delta n \neq 0}^{2} g_{\text {se }}\left(\frac{3 k T n_{l, r}^{*}{ }^{3}}{4 E_{\mathrm{H}} z_{r}^{2}}\right) \\
& +R_{u u^{\prime}}^{2} \underbrace{}_{\substack{\Delta n=0 \\
\ell_{u^{\prime}}=\ell_{u}+1}} \tilde{g}_{\mathrm{se}}\left(\frac{3 k T}{2 \Delta E_{u u^{\prime}}}\right) \\
& +\left.R_{u u^{\prime}}^{2}\right|_{\substack{\Delta n=0 \\
\ell_{u^{\prime}}=\ell_{u}-1}} \tilde{g}_{\text {se }}\left(\frac{3 k T}{2 \Delta E_{u u^{\prime}}}\right) \\
& +R_{l l^{\prime}}^{2} \underbrace{2}_{\substack{\Delta n=0 \\
l_{l^{\prime}}=\ell_{l}+1}} \tilde{g}_{\mathrm{se}}\left(\frac{3 k T}{2 \Delta E_{l l^{\prime}}}\right) \\
& +R_{l l^{\prime}}^{2} \underbrace{2}_{\substack{\Delta n=0 \\
\ell_{l^{\prime}}=\ell_{l}-1}} \tilde{g}_{\mathrm{se}}\left(\frac{3 k T}{2 \Delta E_{l l^{\prime}}}\right)]
\end{aligned}
$$

where

$$
\tilde{g}_{\mathrm{se}}(x)=0.7-1.1 / z_{r}+g_{\mathrm{se}}(x)
$$

is a modified effective Gaunt factor.

In order to use the above formulae, the coordinate matrix elements $R_{a b}$ have to be evaluated. For spectral lines with unknown transition probability, the coordinate matrix elements can be calculated using expressions based on the Coulomb approximation [20]. As the analysis includes only spectral lines with known transition probability the expression [43]

$$
R_{a b}^{2}=\frac{3 h E_{\mathrm{H}}^{2}}{2 \pi \alpha_{\mathrm{f}}^{3} \Delta E_{a b}^{3}}\left\{\begin{aligned}
\frac{g_{a}}{g_{b}} A_{a b} & \text { for } E_{a}>E_{b} \\
A_{b a} & \text { for } E_{a}<E_{b}
\end{aligned}\right.
$$

is used.

\subsection{Basic data and numerical procedure}

Comparison of the NIST [45] and the Kurucz [46] compilation of spectral lines showed that the first contains for all species except $\mathrm{Fe}^{+}$a higher number of energy levels while the second contains for each species a higher number of lines. Consequently, the composition calculations and the bound-free emission coefficients are based on the NIST data while the line emission coefficients are based on the Kurucz data.

The calculated electron Stark widths and the net emission coefficients are based on different subsets of lines from the Kurucz data base. In the calculation of the electron Stark widths the sum over the lines connected to upper or lower level of the considered line, e.g. (36), was restricted to lines with transition wavelength $\lambda_{u l}$ below $10000 \mathrm{~nm}$. The total number of these lines is close to the number of lines used by Menart et 
Table 2. Resulting scaling factors $s_{r}$ for Stark widths, number of internal states $N_{\text {state }}$ and number of spectral lines $N_{\text {line }}$ extracted from [45] and [46], respectively, and number of spectral lines $N_{\text {line }}^{\mathrm{MM}}$ used in [10].

\begin{tabular}{llllll}
\hline & $s_{r}$ & $\Delta s_{r}$ & $N_{\text {state }}$ & $N_{\text {line }}$ & $N_{\text {line }}^{\mathrm{MM}}$ \\
\hline $\mathrm{Ar}$ & 0.47 & 0.27 & 497 & 3552 & 3553 \\
$\mathrm{Ar}^{+}$ & 1.65 & 0.90 & 417 & 6982 & 6959 \\
$\mathrm{Ar}^{2+}$ & 1.0 & & 113 & 1008 & 996 \\
$\mathrm{Fe}$ & 3.21 & 1.59 & 492 & 16739 & 15521 \\
$\mathrm{Fe}^{+}$ & 1.51 & 0.26 & 575 & 40180 & 41644 \\
$\mathrm{Fe}^{2+}$ & 1.0 & & 564 & 22409 & 22243 \\
\hline
\end{tabular}

al [10] (table 2). The present net emission calculations include 5000 lines for each species. These were those lines with the highest line integrated emission coefficient. For comparison with earlier results the integral over wavelength in (3) was restricted to the range given in the corresponding reference. The wavelength integral in (3) was treated by Simpson integration using an inhomogeneous mesh. For each line a mesh was created so that the line integrated emission coefficient was reproduced with an accuracy better than $1 \%$. The meshes of all lines were superimposed on an additional homogeneous mesh with a wavelength step of $1 \mathrm{~nm}$. Further mesh points were introduced at the cutoff wavelengths in the bound-free emission coefficients.

Following Lowke [3] $\mathrm{G}_{1}(x)$ is evaluated by numerical integration of (4). Numerical integration was performed for arguments between $10^{-3}$ and 500 with a relative error of $1 \times 10^{-6}$. Outside this interval $\mathrm{G}_{1}(x)$ is evaluated via its asymptotic expressions.

\section{Results}

\subsection{Emission coefficients of pure argon and iron}

Figures 2 and 1 show the composition of argon and iron plasmas at a pressure of $0.1 \mathrm{MPa}$, respectively, and the wavelength integrated contributions to the emission coefficient of bound-bound, bound-free, and free-free transitions for each radiating species. For all but high temperatures the emission coefficient of iron is several orders of magnitude higher than the emission coefficient of argon. This excess of iron radiation is caused by the higher number of lines of Fe and their lower upper level energies leading to higher upper state population densities. Note that the ionization energy of $\mathrm{Fe}(7.90 \mathrm{eV})$ is lower than the energy of the first excited state of $\mathrm{Ar}(11.55 \mathrm{eV})$. For temperatures above $25000 \mathrm{~K}$, where ions dominate the composition, both emission coefficients are of the same order of magnitude.

The figures show that the total emission coefficient of the plasma is dominated by line radiation. The temperature dependence of the different contributions can be understood considering the plasma composition. The temperature interval where a specific species dominates the line radiation is accompanied by a corresponding 
temperature interval where this species has the highest density. The total emission of each individual species is dominated by line radiation at low temperatures and by bound-free radiation at high temperatures. This behaviour is caused by ionization which decreases the radiator density and increases the density of the radiator ion with increasing temperature. Note however that at the temperatures where the shift from line to bound-free radiation occurs, e.g. $14000 \mathrm{~K}$ for Fe (figure 2), the total emission is dominated by the emission of the ion of the next ionization step. For argon corresponding temperatures are above $30000 \mathrm{~K}$ (figure 1).

Figure 3 shows a comparison of the emission coefficient of an argon plasma at different pressures with values given by Menart et al [19] and measurements done by Evans et al [47]. The wavelength integration covers here the interval from 200 to $3500 \mathrm{~nm}$ corresponding to the sensitivity of the experiment [47]. Both numerical results are identical to within $10 \%$ and lie slightly above the experimental results. The optical path in the measurements includes windows made of vycor. The transmissivity of this material decreases rapidly for wavelengths below $280 \mathrm{~nm}$. Menart et al [19] argue that the difference between the experimental values and their calculation results may be caused by an incomplete compensation of this reduced transmissivity.

\subsection{Electron Stark width scaling factors}

At atmospheric pressure van der Waals broadening is the strongest broadening mechanims only at low temperatures. With increasing temperature first Doppler broadening and then, with the onset of ionization, electron Stark broadening become the dominant broadening mechanism. At a given temperature the line-to-line differences of the electron Stark widths cover approximately two orders of magnitude. Thus the temperatures, where Stark broadening becomes the dominant broadening mechanism, depends on the considered line. While the emission coefficient is independent of the line widths the net emission coefficient for non-zero radius $R$ depends on the absolute line widths and thus on the electron Stark width scaling factors $s_{r}$ introduced in $(36),(38)$, and (39).

The electron Stark width scaling factors $s_{r}$ for $\mathrm{Ar}, \mathrm{Ar}^{+}, \mathrm{Fe}$ and $\mathrm{Fe}^{+}$used for the net emission coefficients presented below are determined by comparison of measured Stark widths with widths calculated here. The employed scaling factors are the mean ratios $s_{r}=\left\langle\Delta \lambda_{\mathrm{Se} \text { exp }} / \Delta \lambda_{\mathrm{Se}}\right\rangle$ where the average is taken over all experimental line widths available for species $r$. This procedure was applied by Menart et al to argon and copper lines [19]. The experimental Stark widths must be specified together with independently measured electron density $N_{\mathrm{e}}$ and temperature $T$. Appropriate values are taken from the reviews given by Konjević et al [48] and Lesage [49] containing measurements for 64, 210, 12 and 14 lines for $\mathrm{Ar}, \mathrm{Ar}^{+}, \mathrm{Fe}$ and $\mathrm{Fe}^{+}$, respectively. The reviews summarize results from dedicated experiments in a temperature range from 6000 to $43000 \mathrm{~K}$. As electron Stark widths for $\mathrm{Ar}^{2+}$ and $\mathrm{Fe}^{2+}$ are not given, the corresponding scaling factors are set to unity in this work. 
Figure 4 shows the individual ratios $\Delta \lambda_{\mathrm{Se}, \exp } / \Delta \lambda_{\mathrm{Se}}$ for $\mathrm{Fe}$ and $\mathrm{Fe}^{+}$lines given by Konjević et al [48], the resulting electron Stark width scaling factors $s_{r}$, and their standard deviation $\Delta s_{r}$. The provided Stark widths were measured on lines in the visible spectral range lying in regions covering approximately 6 and $30 \mathrm{~nm}$ for Fe and $\mathrm{Fe}^{+}$, respectively, except for three measurements given in [48] and the one measurement given in [49] for lines of neutral iron with wavelengths below $426 \mathrm{~nm}$. The Stark width measurements for lines of $\mathrm{Ar}$ and $\mathrm{Ar}^{+}$cover wider ranges from 104 to $850 \mathrm{~nm}$ and from 250 to $720 \mathrm{~nm}$, respectively. The resulting scaling factors have values between 0.48 for Ar and 3.21 for Fe. They are summarized in table 2. In expressions (36), (38), and (39) all transitions connected to upper or lower level of the considered line have a positive contribution to the electron Stark width. Thus, values of $s_{r}$ above unity as those for $\mathrm{Ar}^{+}, \mathrm{Fe}$, and $\mathrm{Fe}^{+}$may be caused by a lack of lines in the underlying data. The scaling factor for Ar is smaller than unity because the employed theory on average overestimates the Stark width of Ar lines. The scaling factor $s_{\mathrm{Ar}}=0.32 \pm 0.14$ determined by Menart et al [19] does not differ significantly from the present value.

Figure 5 shows a comparison of the temperature dependency of the normalized electron Stark width $\Delta \lambda_{\mathrm{Se}} / N_{\mathrm{e}}$ deduced from experiments and calculated here for the neutral iron line at $538.337 \mathrm{~nm}$. Additionally, an experimental fit of the electron Stark width of this line $\Delta \lambda_{\mathrm{Se}} / N_{\mathrm{e}}=1.324 \times 10^{-33} \mathrm{~m}^{4}(T / 13000 \mathrm{~K})^{1.67}$ determined by Zielinska et al [1] is given. The widths calculated here are approximately a factor two below the 'simple theoretical prediction' given by Freudenstein [50]. Figure 5 indicates that for this line both theoretical calculations underestimate the increase of $\Delta \lambda_{\text {Se }} / N_{\mathrm{e}}$ with temperature. A better agreement between theoretical and measured electron Stark widths is obtained when the scaling factor $s_{\mathrm{Fe}}=3.21$ is applied.

\subsection{Net emission coefficients}

This section gives net emission coefficients for argon iron plasmas and comparisons with literature values. The net emission coefficients presented here use the electron Stark width scaling factors summarized in table 2 . The wavelength integral in (3) covers the range from 30 to $2500 \mathrm{~nm}$ unless noted otherwise.

Figure 6 shows the net emission coefficient of argon plasmas at a pressure of $0.1 \mathrm{MPa}$ for cylindrical geometry and values for spherical geometry given by Menart et al [10]. The net emission coefficient rapidly increases with increasing temperature and decreases with growing plasma radius. The agreement of both net emission coefficients is better than approximately $10 \%$.

Figure 7 shows net emission coefficients of iron plasmas at a pressure of $0.1 \mathrm{MPa}$ for cylindrical and for spherical geometry calculated here and values for spherical geometry given by Menart et al [10]. It shows that the net emission coefficient for cylindrical geometry is smaller than values for spherical geometry. This is caused by the higher amount of irradiation and absorbed power at the arc axis when cylindrical geometry is considered. The results for spherical geometry are in good agreement with those given by 
Menart et al [10] for all but the highest radius of $1 \mathrm{~m}$. The difference between literature values and the present results in spherical geometry may be caused by deviations in the number of considered lines, transition probabilities and Stark widths. The present results for cylindrical geometry also show good agreement with the literature values for radii below $10^{-3} \mathrm{~m}$. For larger radii they show a faster decrease with increasing radius.

Figure 8 shows the influence of the electron Stark widths scaling factors $s_{r}$ on the net emissions cofficients of argon and iron plasmas at a pressure of $0.1 \mathrm{MPa}$ in cylindrical geometry. The figure displays contour lines of $\epsilon_{\text {net }} / \epsilon_{\text {net }}\left(s_{r}=1\right)-1$ where $\epsilon_{\text {net }}\left(s_{r}=1\right)$ is the net emission coefficient calculated with $s_{r}=1$ for all species. It shows that the scaling factors have no influence on $\epsilon_{\text {net }}$ at low temperatures where Stark broadening is not the dominant broadening mechanism. For argon this region extends to higher temperatures than for iron, because iron has a smaller ionization potential. The influence of the electron Stark widths is small for small plasma radii because here the plasma remains optically thin for the emission lines. There is an intermediate range of radii where a scaling factor above unity leads to an increase of $\epsilon_{\text {net }}$ and a scaling factor below unity to a decrease of $\epsilon_{\text {net. }}$. In this intermediate range the net emission coefficient is dominated by the emission near the line centres. Enlarged line widths lead to reduced absorption coefficients near the line centres so that their contribution to $\epsilon_{\text {net }}$ increases. In the line wings an increase of the line width leads to higher optical depths and a decrease of their contribution to $\epsilon_{\text {net }}$. With increasing plasma radius the optical depth near the line centre increases faster than in the line wings. Thus, when the line centres have reached high optical depth the influence of $s_{r}$ is weakened first and reversed in the limit of high plasma radius. The opposing behaviours of the absorption coefficient in the line centres and the line wings and the restriction of the influence of $s_{r}$ to the temperature region where species $r$ has a high density lead to the formation of maxima and minima in $\epsilon_{\text {net }} / \epsilon_{\text {net }}\left(s_{r}=1\right)-1$. For the employed scaling factors these extrema are -17 and $12 \%$ for argon and 11 and $37 \%$ for iron. In the typical parameter range of GMAW processes $\left(T=5000-10000 \mathrm{~K}, R=1-2 \times 10^{-3} \mathrm{~m}\right)[1,2]$ the scaling factors $s_{r}$ lead to an increase of $\epsilon_{\text {net }}$ of an iron plasma by between 3 and $22 \%$.

The standard deviations $\Delta s_{r}$ of the electron Stark width scaling factors give rise to an uncertainty $\Delta \epsilon_{\text {net }}$ of the net emission coefficient. The variation of this uncertainty as a function of plasma temperature and radius has the same structure as the influence of the scaling factors on $\epsilon_{\text {net }}$ shown in figure 8 . The maximum absolute values of $\Delta \epsilon_{\text {net }}$ in the intermediate range of radii are 12 and $14 \%$ for argon, and 4 and $13 \%$ for iron plasmas. In the GMAW range of parameters the uncertainty for iron plasmas has values between 0 and $8 \%$.

For the radius $R=1 \mathrm{~m}$ the net emission coefficients given by Menart et al [10] decrease with increasing temperature for temperatures between approximately 6000 and $9000 \mathrm{~K}$ (figure 7). The present results in cylindrical and spherical geometry both reproduce this behaviour. There is good agreement between the present results for spherical geometry and the literature values for temperatures below $6000 \mathrm{~K}$ and above $13000 \mathrm{~K}$, however at $9000 \mathrm{~K}$ the present results for spherical geometry are a factor of 
approximately 3.3 larger than the literature value. This descrepancy may be caused by differences in the employed Stark widths and continuous emission coefficients. The uncertainty $\Delta \epsilon_{\text {net }}$ of the net emission coefficient of iron has its highest values in the region of the local minimum of $\epsilon_{\text {net. }}$. It is $23 \%$ at $R=1 \mathrm{~m}$ and $T=9000 \mathrm{~K}$. Calculations where the continuous emission coefficient of Fe is increased by a factor of two show a decreases of the local minimum of $\epsilon_{\text {net }}$ by $30 \%$.

Figure 9 shows the influence of the geometry on the net emission coefficient of an iron plasma at a pressure of $0.1 \mathrm{MPa}$. The figure displays contour lines of $\epsilon_{\text {net }}^{\mathrm{cyl}} / \epsilon_{\text {net }}^{\mathrm{sph}}-1$ where $\epsilon_{\text {net }}^{\text {cyl }}$ and $\epsilon_{\text {net }}^{\text {sph }}$ are the net emission coefficient for cylindrical and spherical geometry, respectively. For both geometries the electron Stark widths scaling factors in table 2 have been used. As expected from the geometry functions, the net emission coefficients are smaller for cylindrical geometry than for spherical geometry. For most temperatures the ratio of the net emission coefficient decreases monotonically with increasing plasma radius. The maximum reduction of $47 \%$ induced by a change from spherical to cylindrical geometry in the considered parameter range lies at $T=9500 \mathrm{~K}$ and $R=1 \mathrm{~m}$. In the region relevant for GMAW processes the net emission coefficient of iron plasmas for cylindrical geometry is 30 to $37 \%$ smaller than values for spherical geometry. For argon plasmas the corresponding reduction is only between 5 and $9 \%$.

Figure 10 shows net emission coefficients of argon iron plasmas for different plasma radii and iron fractions from zero to unity for cylindrical geometry and values for spherical geometry given by Menart et al [10]. It shows that for low temperatures the net emission of the plasma is determined by the radiation of iron as soon as small amounts of iron are contained in the plasma. For temperatures below $10000 \mathrm{~K}$ this is the case for iron fractions above $0.01 \%$. The differences between the values calculated here and in the literature [10] seen in figure 7 reappear in figure 10. While the agreement between both results is good at small plasma radii the values calculated here decrease faster with plasma radius than those given by Menart et al [10].

Figure 11 shows the contributions to the net emission coefficient for cylindrical geometry in the wavelength intervals 30-200 nm, 200-700 nm and 700-2500 nm representing the ultraviolet (UV), the visible and the infrared spectral range (IR), respectively. The net emission coefficients for $R=0$ and $0.001 \mathrm{~m}$ are compared. The main contribution to the net emission coefficient comes from the visible spectral range for low temperatures and from the UV for high temperatures. The temperature where the shift between the spectral ranges occurs depends on the plasma radius and the iron mole fraction. At low temperatures the infrared contributes a higher fraction to the net emission coefficient than the ultraviolet. Due to the small absorption in the IR spectral range the percentage of the net emission coefficient originating from the infrared increases with increasing plasma radius. For $9000 \mathrm{~K}$ this fraction increases from 3 to $22 \%$ when for radius increases from 0.001 to $0.01 \mathrm{~m}$. 


\section{Conclusions}

The net emission coefficient increases rapidly with increasing temperature and is dominated by the line radiation of iron, as soon as small amounts of iron are contained in the plasma. The net emission coefficient is dominated by radiation from the visible spectral range at low temperatures and dominated by radiation from the ultraviolet at high temperatures. The infrared radiation exceeds the ultraviolet only at low temperatures and its fraction of net emission coefficient increases with increasing plasma radius. The net emission coefficients for argon iron plasmas at atmospheric pressure calculated here are in good agreement with values from earlier investigations [10], even though the number of lines taken into account here is with 5000 lines per species considerably smaller. The semi-empirical Stark broadening theory [48] was employed to calculate the Stark widths of the spectral lines. Additionally, the widths of the lines of $\mathrm{Ar}, \mathrm{Ar}^{+}, \mathrm{Fe}$ and $\mathrm{Fe}^{+}$were scaled to experimental electron Stark widths. For temperatures and plasma radii typical of the iron containing core region of arcs occurring in the gas metal arc welding process $\left(T=5000-10000 \mathrm{~K}, R=1-2 \times 10^{-3} \mathrm{~m}\right)[1,2]$ this scaling procedure leads to an increase of the net emission coefficient by between 2 and $23 \%$. The net emission coefficients of argon iron plasmas for cylindrical geometry presented in this work decrease faster with increasing radius $R$ than earlier results where spherical geometry is considered. In the GMAW range of radii and temperatures the net emission coefficient of iron plasmas for cylindrical geometry is between 30 and $37 \%$ smaller than corresponding values calculated for spherical geometry.

\section{Acknowledgment}

This work was funded by the Deutsche Forschungsgemeinschaft (DFG) under grants UH 106/3-1. The author would like to thank H. Schneidenbach and D. Uhrlandt for helpful suggestions.

\section{References}

[1] Zielinska S, Musiol K, Dzierzega K, Pellerin S, Valensi F, de Izarra C and Briand F 2010 Investigations of GMAW plasma by optical emission spectroscopy Plasma Sources Sci. Technol. $16832-8$

[2] Rouffet M E, Wendt M, Goett G, Kozakov R, Schoepp H, Weltmann K D and Uhrlandt D 2010 Spectroscopic investiagtion of the high-current phase of a pulsed GMAW process J. Phys. D: Appl. Phys. 43434003

[3] Lowke J J 1974 Predictions of arc temperature profiles using approximate emission coefficients for radiation losses J. Quant. Spectrosc. Radiat. Transfer 14 111-22

[4] Lowke J J, Tanaka M and Murphy A B 2009 Metal vapours in MIG arcs cause (1) minima in central arc temperatures and (2) increased arc voltages 62nd Annual Assembly of Int. Inst. Welding (IIW) IIW Doc.212-1139-09

[5] Schnick M, Fuessel U, Hertel M, Spille-Kohoff A and Murphy A B 2010 Metal vapour causes a central minimum in arc temperature in gas-metal arc welding through increased radiative emission J. Phys. D: Appl. Phys. 43022001 
[6] Modest M F 2003 Radiative heat transfer (Amsterdam: Academic Press)

[7] Cram L E 1985 Statistical evaluation of raditive power losses from thermal plasmas due to spectral lines J. Phys. D: Appl. Phys. 18 401-11

[8] Essoltani A, Proulx P, Boulos M I and Gleizes A 1990 Radiation and self-absorption in arong-iron plasmas at atmospheric pressure J. Analyt. Atomic Spectromet. 5 543-7

[9] Gleizes A, Gonzalez J J, Liani B and Raynal G 1993 Calculation of net emission coefficient of thermal plasmas in mixtures of gas with metallic vapour J. Phys. D: Appl. Phys. 26 1921-7

[10] Menart J and Malik S 2002 Net emission coefficients for argon-iron thermal plasmas J. Phys. D: Appl. Phys. 35 867-74

[11] Moscicki T, Hoffman J and Szyanski Z 2008 Net emission coefficients of low temperature thermal iron-helium plasma Optica Applicata 38 365-73

[12] Allen C W 1966 Statistical spectroscopy Mon. Not. R. Astron. Soc. 133 21-46

[13] Drawin H W and Emard F 1973 Optical escape factors for bound-bound and free-bound radiation from plasmas. I. Constant source function Beitr. Plasmaphys. 13 143-68

[14] Irons F E 1979 Escape factors in plasma spectroscopy .1. Escape factors defined and evaluated $J$. Quant. Spectrosc. Radiat. Transfer 22 1-20

[15] Kurucz R L and Peytremann E 1975 A table of semi-empirical gf values Smithonian Astrophysical Observatory Special Report 362 (NASA-CR-142879)

[16] Bautista M A 1997 Atomic data from the iron project - XX. Photoionization cross sections and oscillator strengths for Fe I Astron. Astrophys. Suppl. Series 122 167-76

[17] Nahar S N 1995 Atomic data from the iron project - VII. Radiative dipole transition probabilities for Fe II Astron. Astrophys. 293 967-77

[18] Traving G 1968 in Lochte-Holtgreven W Plasma diagnostics (New York: AIP Press) p. 66

[19] Menart J, Heberlein J and Pfender E 1996 Line-by-line method of calculating emission ceofficients for thermal plasmas consisting of monatomic species J. Quant. Spectrosc. Radiat. Transfer $\mathbf{5 6}$ 377-398

[20] Griem H R 1968 Semiempirical formulas for the electron-impact widths and shifts of isolated ion lines in plasmas Phys. Rev. 165 258-66

[21] Dimitrijević M S and Konjević N 1986 Simple formulae for estimating Stark widths and shifts of neutral atom lines Astron. Astrophys. 163 297-300

[22] Dimitrijević M S and Konjević N 1980 Stark widths of doubly- and triply-ionized atom lines $J$. Quant. Spectrosc. Radiat. Transfer 24 451-59

[23] Church C H, Liberman I, Schlecht R G and Swanson B W 1966 Studies of highly radiative plasmas using the wall-stabilzed pulsed arc discharge AIAA Jour. 4 1947-53

[24] Abramowitz M, Stegun I A 1972 Handbook of mathematical functions (New York: Dover Publications)

[25] Lee H E and Cram L E 1984 On the theory of radiation-dominated wall-stabilised arcs J. Phys. D: Appl. Phys. 18 1561-73

[26] Ebeling W, Kraeft W-D and Kremp D 1976 Ergebnisse der Plasmaphysik und der Gaselelectronik Band 5 (Berlin: Akademie-Verlag)

[27] Richter J in Plasma Diagnostics ed. Lochte-Holtgreven (New York: AIP Press) p. 1

[28] Aubrecht V, Bartlova M and Bogatyreva N 2009 29th ICPIG July 12-17, Cancún, Mexiko Radiation properties of argon thermal plasma in various spectral regions

[29] Seaton M J 1987 Atomic data for opacity calculations: I. General description J. Phys. B: At. Mol. Phys. 20 6363-78

[30] Verner D A, Ferland G J, Korista K T and Yakovlev D G 1996 Atomic data for astrophysics II. New analytic fits for photoionization cross sections of atoms and ions Astrophys. Jour. 465 $487-98$

[31] Marr G V and West J B 1976 Absolute photoionization cross-section tables for helium, neon, argon, and krypton in the VUV spectral regions Atomic Data and Nuclear Data Tables 18 497-508

[32] Bautista M A and Pradhan A K 1995 Photoionization of neutral iron J. Phys. B: At. Mol. Opt. 
Phys. 28 L173-9

[33] Nahar S N and Pradhan A K 1994 Atomic data for opacity calculations: XX. Photoionization cross sections and oscillator strengths for Fe II J. Phys. B: At. Mol. Opt. Phys. 27 429-46

[34] Cunto W, Mendoza C, Ochsenbein F and Zeippen C J 1993 TOPbase at the CDS Astron. Astrophys. 275 L5-8

[35] Verner D A, Yakolev D G, Band I M and Trzhaskovskaya M B 1993 Subshell photoionization cross sections and ionization energies of atoms and ions from He to Zn Atomic Data and Nuclear Data Tables 55 233-80

[36] Brum K T A L 2004 Contiuum radiation in a high pressure argon-mercury lamp Plasma Sources Sci. Technol. 13 387-94

[37] Kasýanov V and Starostin A 1965 On theory of bremsstrahlung of slow electrons on atoms Sov. Phys. JETP 21193

[38] Suzuki M, Taniguchi T and Tagashira H 1990 Momentum transfer cross section of argon deduced from electron drift velocity data J. Phys. D: Appl. Phys. 23 842-50

[39] Kihara T, Taylor M H and Hirschfelder J O 1960 Transport properties for gases assuming inverse power intermolecular potentials Phys. Fluids 3 715-20

[40] Lide D R 2005 CRC Handbook of Chemistry and Physics (Boca Raton: CRC Press)

[41] Whiting E E 1968 An empirical approximation to the Voigt profile J. Quant. Spectrosc. Radiat. Transfer 8 1379-84

[42] Unsöld A 1968 Physik der Sternatmosphren 2nd ed. (Berlin: Springer Verlag)

[43] Griem H R 1974 Spectral line broadening by plasmas (New York, NY: Academic Press)

[44] Griem H R, Baranger M, Kolb A C and Oertel G 1962 Stark broadening on neutral helium lines in a plasma Phys. Rev. 125 177-95

[45] Ralchenko Yu, Kramida A E, Reader J and NIST ASD Team 2008 NIST Atomic Spectra Database (version 3.1.5) (Gaithersburg, MD: National Inst. Standards and Tech.) http://physics.nist.$\operatorname{gov} / \operatorname{asd} 3$

[46] Kurucz R L and Bell B 1995 Atomic Line Data, Kurucz CD-ROM No. 23 (Cambridge, Mass.: Smithsonian Astrophysical Observatory) http://www.cfa.harvard.edu/amp/ampdata/kurucz23/sekur.html

[47] Evans D L and Tankin R S 1967 Measurement of emission and absorption of radiation by an argon plasma Phys. Fluids B 2 1137-44

[48] Konjević N, Lesage A, Fuhr J R and Wiese W L 2002 Experimental Stark widths and shifts for spectral lines of neutral and ionized atoms (A critcal review of selected data for the period 1989 through 2000) J. Phys. Chem. Ref. Data 31 819-927

[49] Lesage A 2009 Experimental Stark widths and shifts for spectral lines of neutral and ionized atoms A critcal review of selected data for the period 2001-2007 New Astronomy Reviews52 471-535

[50] Freudenstein S and Cooper J 1979 Stark broadening of Fe I $5383 \AA$ Astron. Astrophys. 71 283-88 

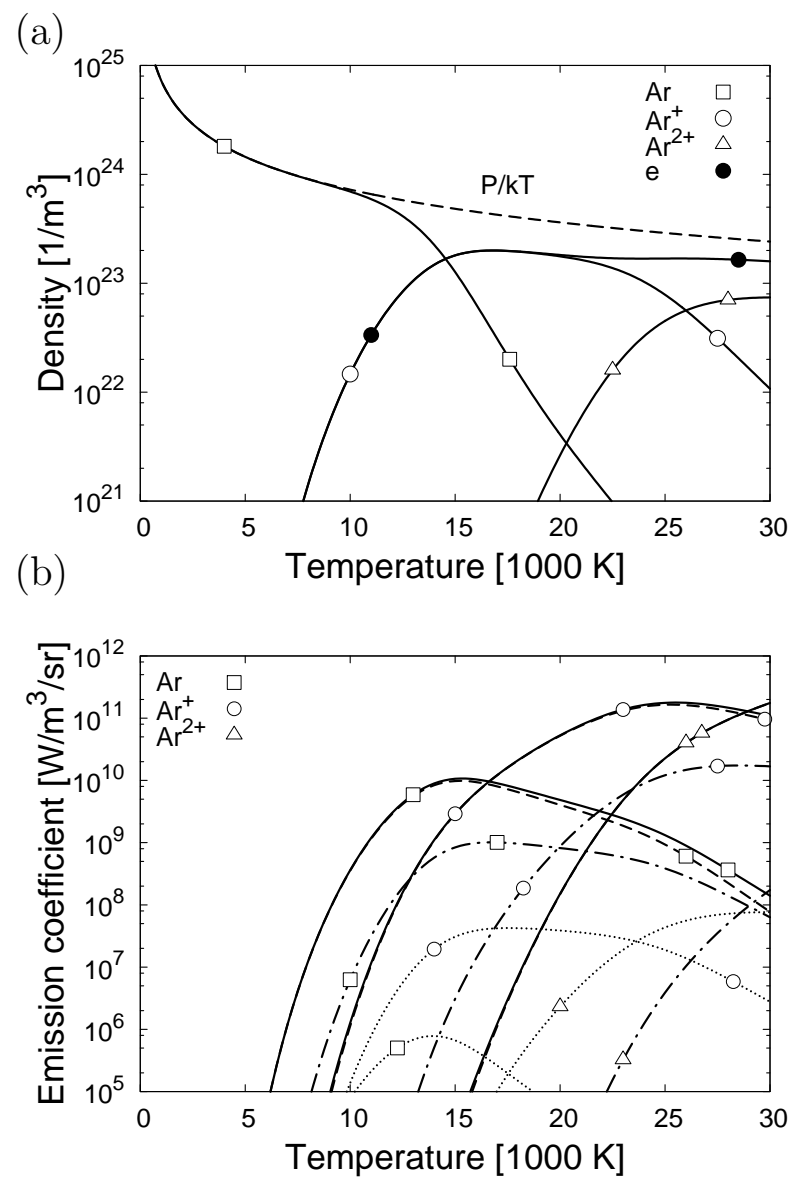

Figure 1. (a) Composition of an argon plasma at $p=0.1 \mathrm{MPa}$ and (b) contributions of bound-bound $(---)$, bound-free $(-\cdot-)$ and free-free $(\ldots \ldots)$ transitions to the emission coefficient and their sum $(-)$. 

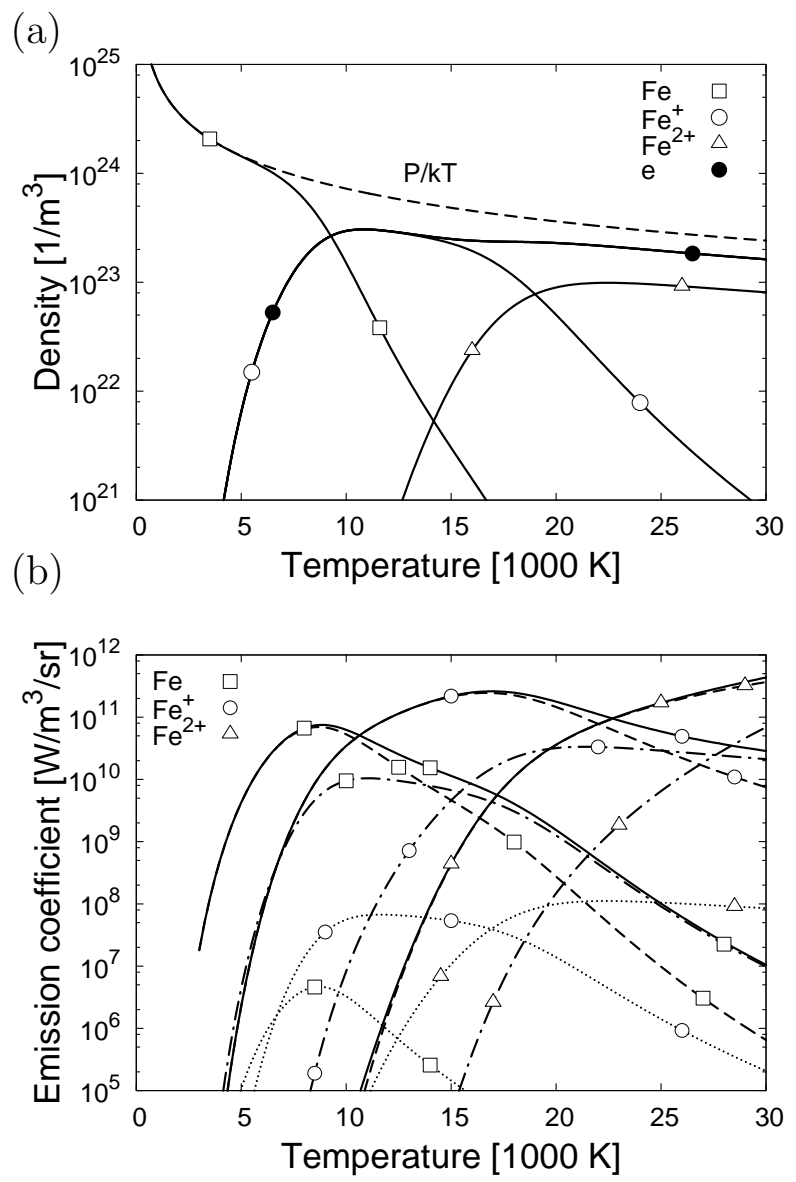

Figure 2. (a) Composition of an iron plasma at $p=0.1 \mathrm{MPa}$ and (b) contributions of bound-bound $(---)$, bound-free $(-\cdot-)$ and free-free $(\ldots \ldots)$ transitions to the emission coefficient and their sum $(-)$.

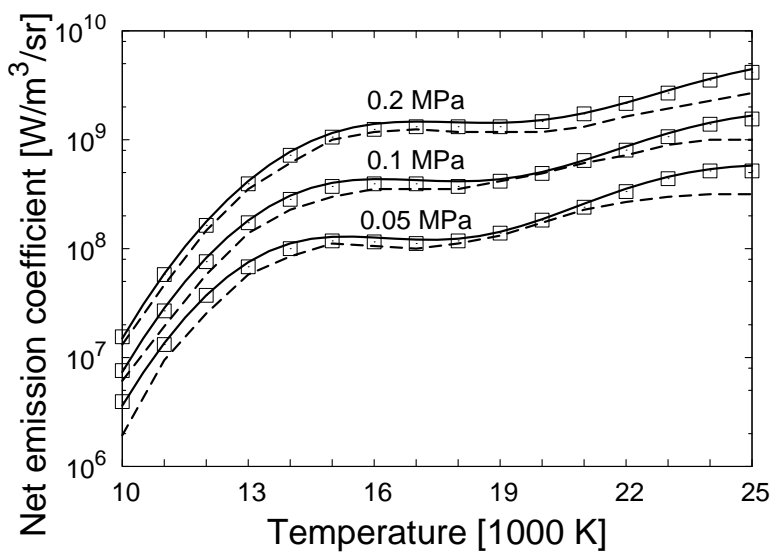

Figure 3. Emission coefficients of argon plasmas in the wavelength interval from 200 to $3500 \mathrm{~nm}$ calculated here $(-)$, in [19] $(\square)$, and measured in [47] (---). 


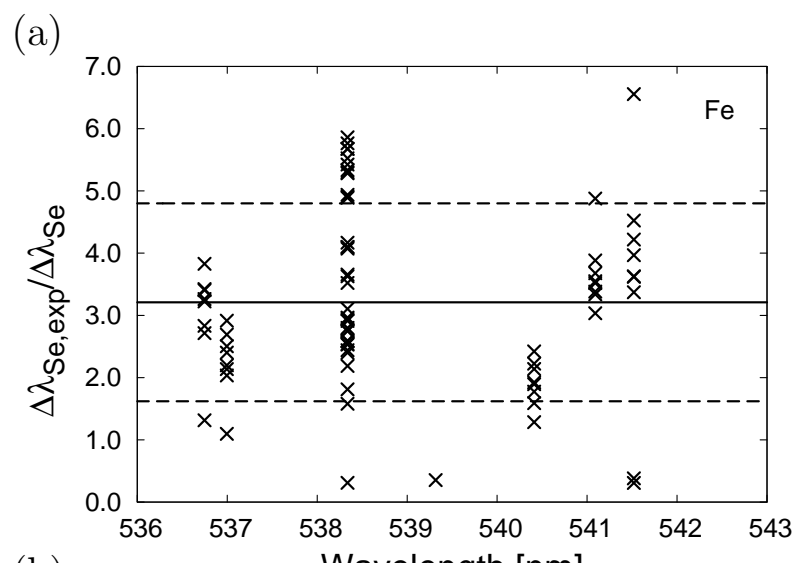

(b)

Wavelength [nm]

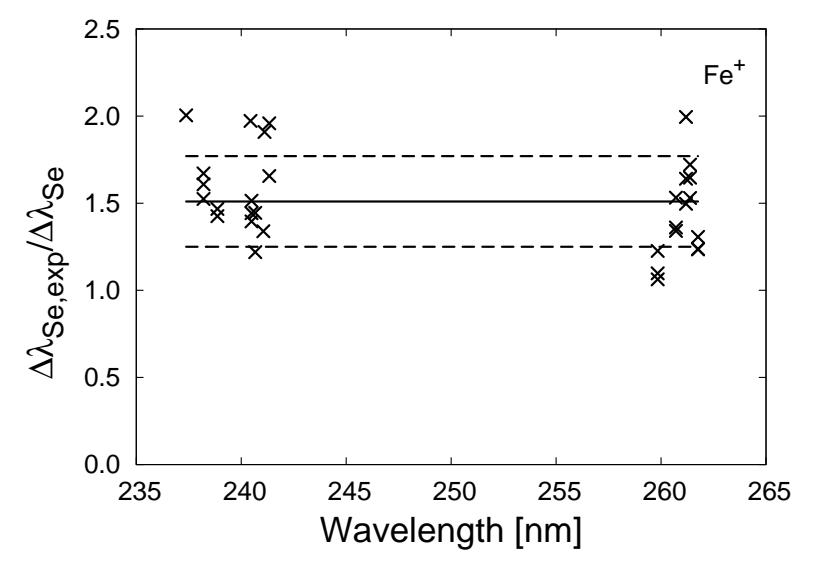

Figure 4. Ratios $\Delta \lambda_{\mathrm{Se}, \exp } / \Delta \lambda_{\mathrm{Se}}$ of experimental and theoretical HWHM electron Stark widths calculated with $s_{r}=1$ for (a) Fe, and (b) $\mathrm{Fe}^{+}$: experimental widths $(\times)$ summarized in [48], mean value $(-)$, and standard deviation $(---)$.

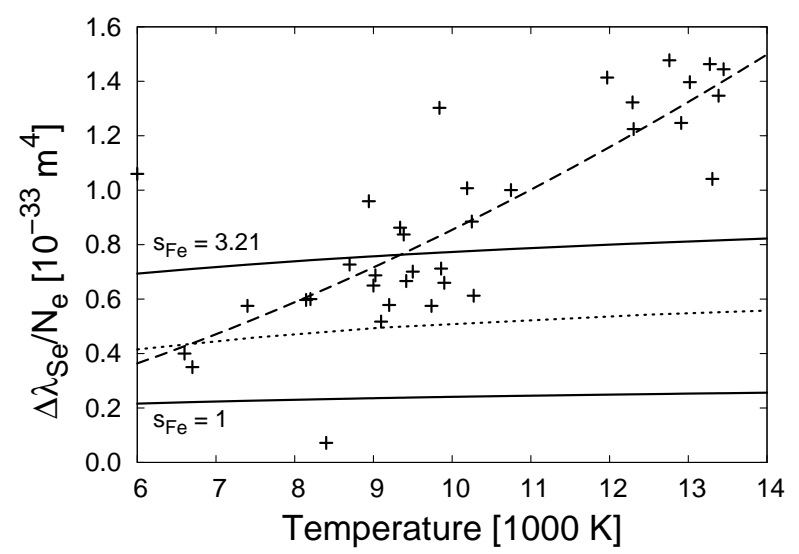

Figure 5. Normalized electron Stark widths $\Delta \lambda_{\mathrm{Se}} / N_{\mathrm{e}}$ of the Fe line at $538.337 \mathrm{~nm}$ : data from $[48](+)$, present results $(-)$ with $s_{\mathrm{Fe}}=1$ and resulting scaling factor $s_{\mathrm{Fe}}=3.21$, 'simple theoretical prediction' from [50] $(\cdots \cdots)$, experimental fit from [1] $(---)$. 


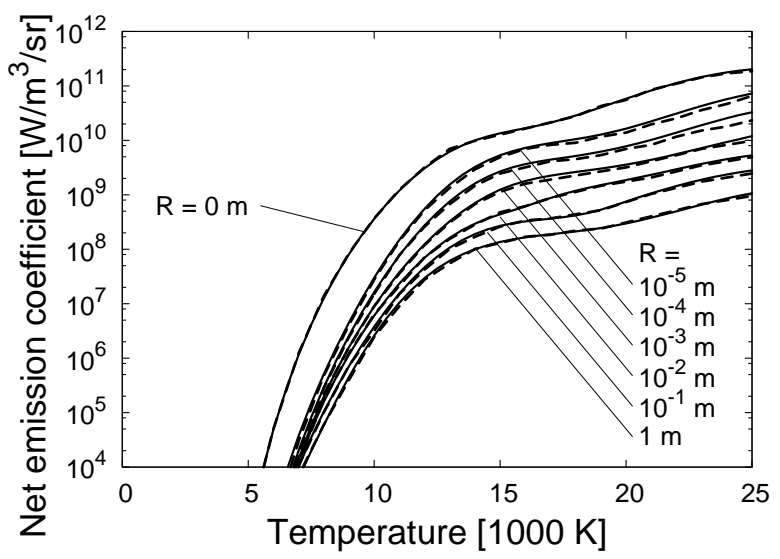

Figure 6. Net emission coefficients of an argon plasma at $P=0.1 \mathrm{MPa}$ : cylindrical geometry from this work $(-)$, spherical geometry from $[10](---)$.

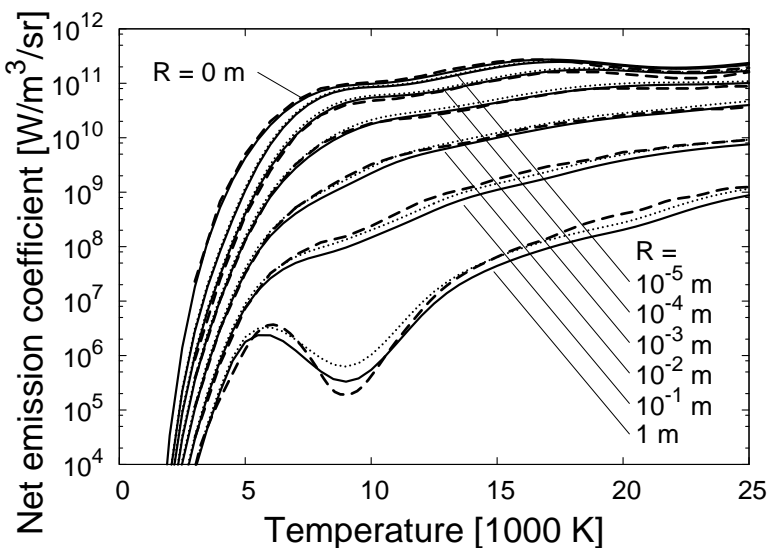

Figure 7. Net emission coefficient of an iron plasma at $P=0.1 \mathrm{MPa}$ : cylindrical $(-)$ and spherical geometry $(\ldots \ldots)$ from this work, spherical geometry from [10] $(--)$. 
(a)

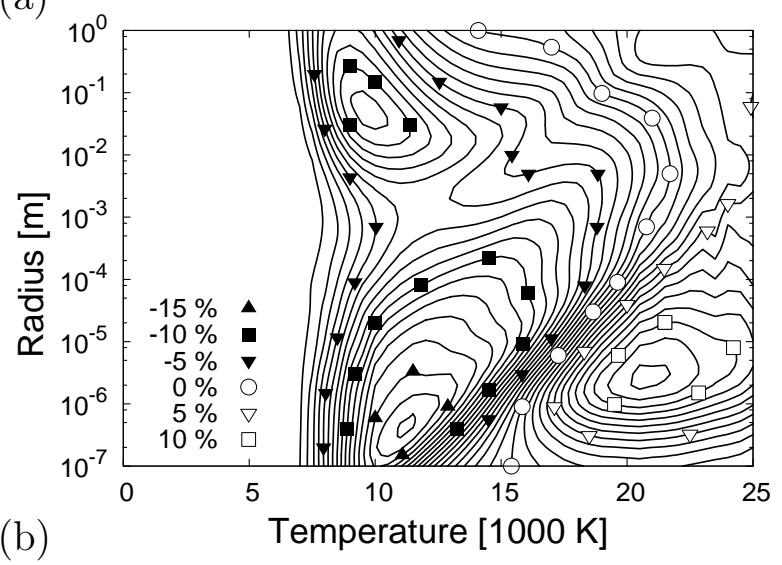

(b)

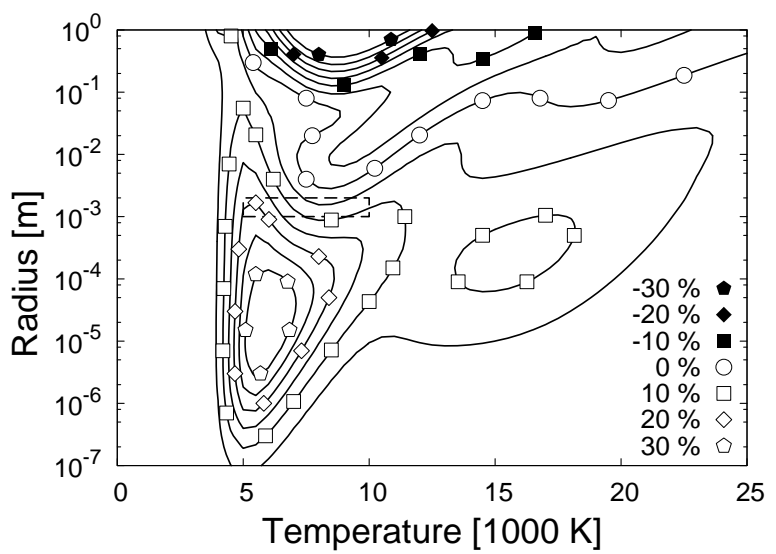

Figure 8. Influence of the scaling factors $s_{r}$ : contour lines of $\epsilon_{\text {net }} / \epsilon_{\text {net }}\left(s_{r}=1\right)-1$ (—) for (a) argon and (b) iron plasmas at $P=0.1 \mathrm{MPa}$ in cylindrical geometry.

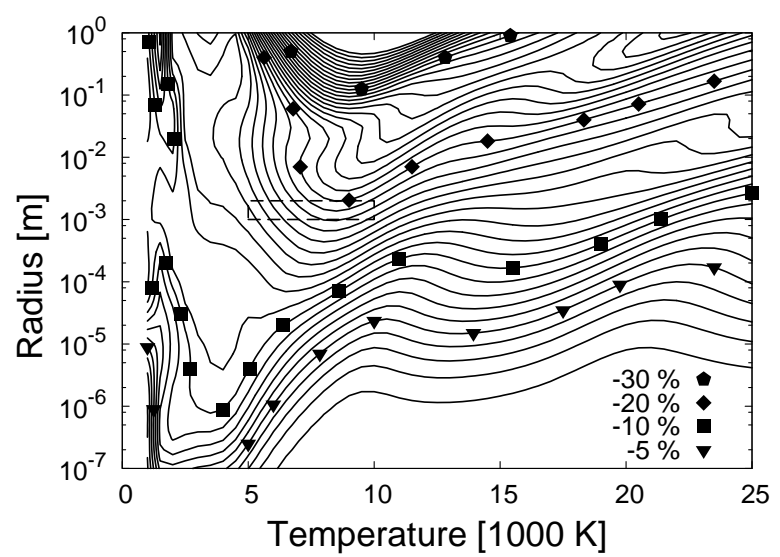

Figure 9. Influence of the considered geometry on the net emission coefficient of an iron plasma at $P=0.1 \mathrm{MPa}$ : contour lines of $\epsilon_{\text {net }}^{\text {cyl }} / \epsilon_{\text {net }}^{\mathrm{sph}}-1(-)$, typical parameter range for GMAW processes (-- ). 
(a)

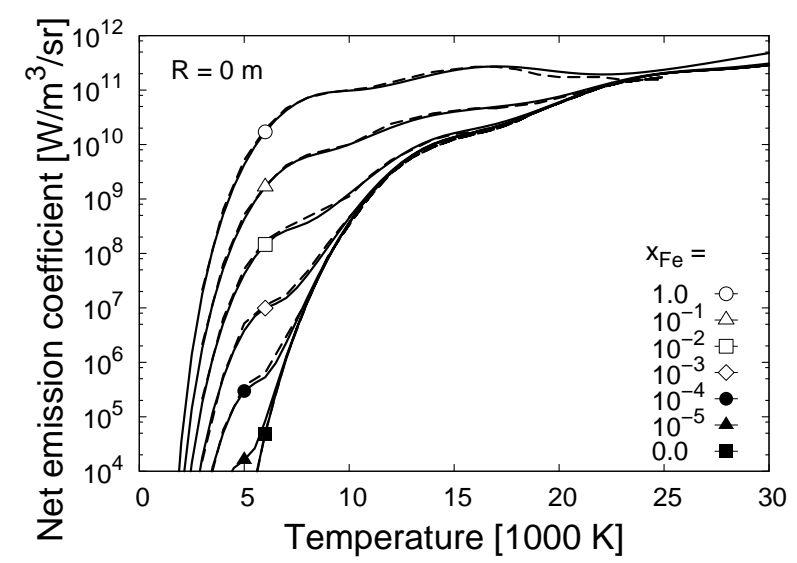

(b)

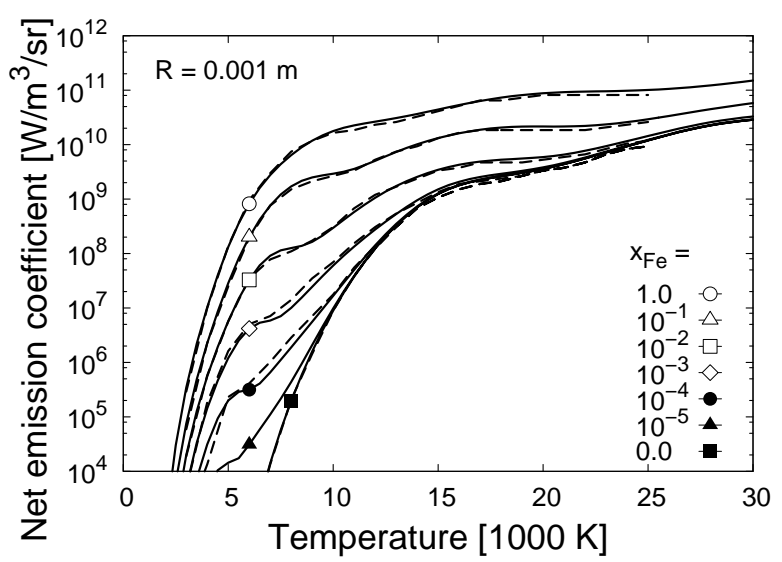

(c)

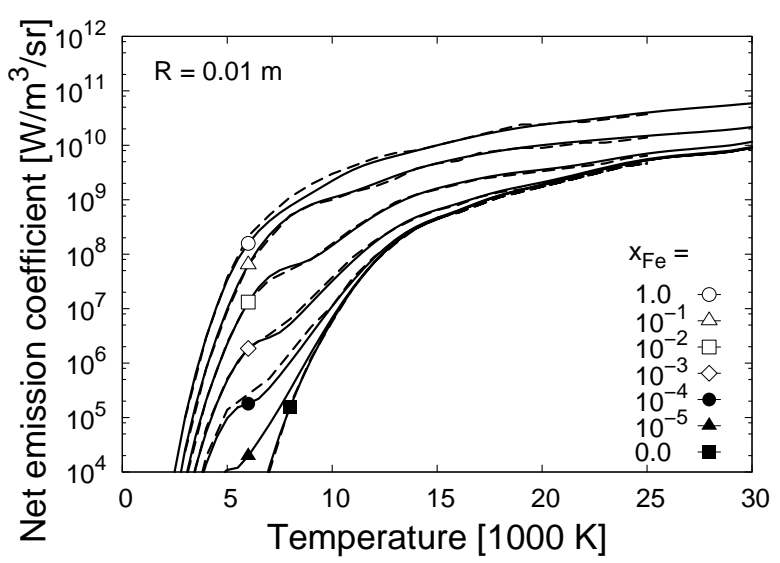

Figure 10. Net emission coefficients of argon iron plasmas at $P=0.1 \mathrm{MPa}$ with iron mole fractions $x_{\mathrm{Fe}}$ from 0 to 1 for (a) $R=0$, (b) $R=1 \times 10^{-3} \mathrm{~m}$, and (c) $R=1 \times 10^{-2} \mathrm{~m}$ : present work for cylindrical geometry (-), results from [10] for spherical geometry $(---)$. 
(a)

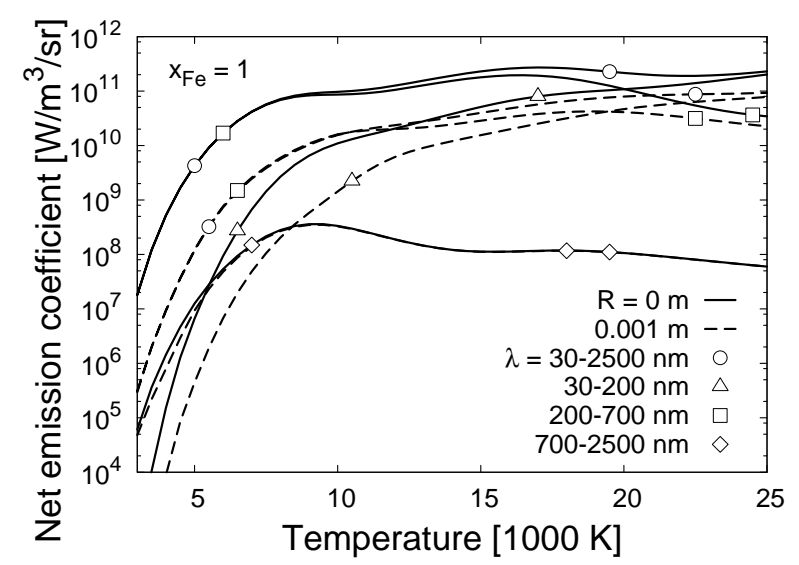

(b)

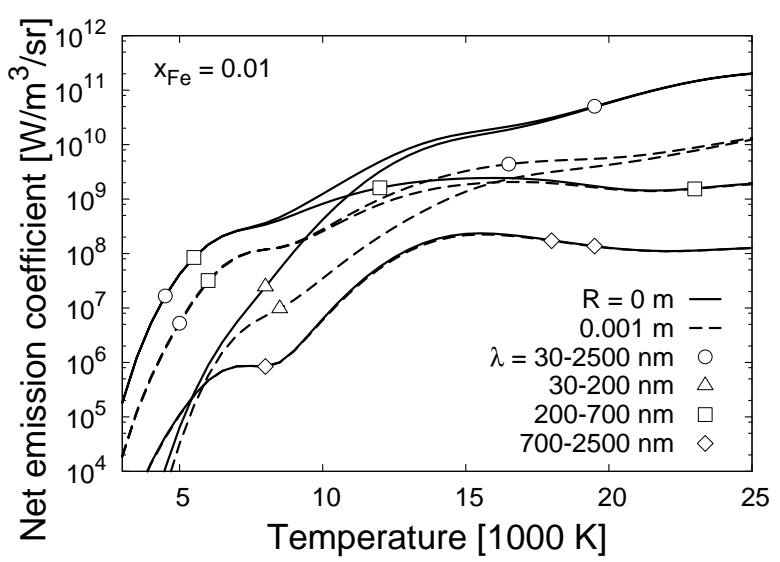

Figure 11. Net emission coefficients for argon iron plasmas at $P=0.1 \mathrm{MPa}$ for cylindrical geometry in different wavelength intervals and iron mole fractions (a) $x_{\mathrm{Fe}}=1$ and (b) $x_{\mathrm{Fe}}=0.01$ : net emission coefficients for radius $R=0(-)$, and $R=0.001 \mathrm{~m}(---)$. 